\title{
Case Report: Isolated Central Nervous System Melioidosis from a Non-Endemic Area
}

\author{
Biswamohan Mishra, ${ }^{1}$ Venugopalan Y. Vishnu, ${ }^{1}$ Rohit Bhatia, ${ }^{1}$ Ajay Garg, ${ }^{2}$ Ramesh Sharanappa Doddamani, ${ }^{3}$ Parul Singh, ${ }^{4}$ \\ Mehar Chand Sharma, ${ }^{5}$ Mamta Bhushan Singh, ${ }^{1}$ Roopa Rajan, ${ }^{1}$ Anu Gupta, ${ }^{1}$ and Madakasira Vasantha Padma Srivastava ${ }^{1 *}$ \\ ${ }^{1}$ Department of Neurology, All India Institute of Medical Sciences, New Delhi, India; ${ }^{2}$ Department of Neuroradiology, All India Institute of Medical \\ Sciences, New Delhi, India; ${ }^{3}$ Department of Neurosurgery, All India Institute of Medical Sciences, New Delhi, India; ${ }^{4}$ Department of Microbiology, \\ All India Institue of Medical Sciences, New Delhi, India; ${ }^{5}$ Department of NeuroPathology, All India Institute of Medical Sciences, New Delhi, India
}

Abstract. Central nervous system (CNS) melioidosis is a rare neurological infectious disease which carries a high mortality. We describe a previously healthy middle-aged female, who presented to us with left-sided hemiparesis and was on antitubercular therapy from a previous presumed diagnosis of CNS tuberculoma. Non-characteristic imaging picture, multiple negative body fluid cultures, and positive Cerebrospinal fluid galactomannan led to a further delay in diagnosis. Gram stain of the tissue obtained from brain biopsy revealed Gram-negative rods in "safety pin" appearance. By picking up the colonies that appeared on blood agar and MacConkey agar, the identification of the clinical isolates was performed using VITEK ${ }^{\circledR}$ matrix (BioMérieux, Marcy-L'Etoile, France)-assisted laser desorption ionization time-of-flight mass spectrometry (VITEK MALDI TOF MS database version 3.2) which revealed Burkholderia pseudomallei. After the institution of appropriate treatment, she survived but with significant morbidity. A high index of suspicion should be kept for such previously healthy individuals belonging to non-endemic areas, where presentation is suspicious of an infective etiology, but not improving despite appropriate therapy. This may help in early recognition and institution of recommended treatment so that mortality can be avoided.

\section{CASE PRESENTATION}

A 28-year-old woman, from Rajasthan, India, without any comorbidities, presented with left hemiparesis for 4 months. Four months back, she was admitted to another hospital with a 1-month history of fever, frontal headache, and intermittent vomiting. Magnetic resonance imaging (MRI) of the brain had shown a well-defined white matter lesion with mass effect in the right parieto-occipital region (Figure 1A-D) suggestive of an infective granuloma probably tubercular. Cerebrospinal fluid (CSF) analysis revealed cell count of 55 (90\% lymphocytes), normal sugars (62 $\mathrm{mg} / \mathrm{dL}$ ), mildly raised protein of 61 $\mathrm{mg} / \mathrm{dL}(\mathrm{N}-15-45 \mathrm{mg} / \mathrm{dL})$, and sterile cultures. Accordingly, antitubercular therapy (ATT) was initiated.

Meanwhile, she complained of left upper and lower limb weakness, and the weakness was maximum (power in the left upper and lower limbs-Medical Research Council [MRC] grade 0/5) in 2 days. A biopsy of the parietal lesion revealed brain parenchyma with multiple abscesses without any mitotic tissue. Cultures were sterile. Levetiracetam was added following multiple episodes of generalized seizures, and ATT was modified. After 2 weeks, she started improving, fever subsided, and seizures abated, and she was able to walk with mild support. For the next 4 months, her clinical condition remained static. She was on isoniazid $300 \mathrm{mg}$, rifampicin $450 \mathrm{mg}$, pyrazinamide $1250 \mathrm{mg}$, ethambutol $800 \mathrm{mg}$, injection streptomycin $750 \mathrm{mg}$, all once daily and levetiracetam $500 \mathrm{mg}$ twice daily (BD) when she first presented to us.

Examination revealed a conscious, oriented patient with left upper motor facial palsy without any meningeal signs. She had power MRC 4-/5 in the left upper and lower limbs with grade 2 spasticity and brisk reflexes and extensor plantar on the left side. Sensations were impaired on the left side, and she walked with a spastic gait with one-person support.

\footnotetext{
*Address correspondence to Madakasira Vasantha Padma Srivastava, Department of Neurology, C. N. Centre, All India Institute of Medical Sciences, Rm. No. 702, New Delhi 110029, India. E-mail: vasanthapadma123@gmail.com
}

In the present case, an infective etiology causing a spaceoccupying lesion in the right frontoparietal cortex was considered first. Although brain abscess, tuberculoma, and neurocysticercosis were the initial differentials, acute disseminated encephalomyelitis and cerebral venous sinus thrombosis were also considered.

Her initial hemogram, and liver and kidney function tests were normal (Supplemental Table S1). Magnetic resonance imaging of the brain showed an increase in the extent to white matter hyperintensity to the right anterior frontal lobe and left corona radiata (Figure $1 \mathrm{E}$ and $\mathrm{F}$ ), with multiple iso-intense foci within T2-hyperintense lesion (Figure 1E) and patchy nodular enhancement in the right frontal lobe and left centrum semiovale (Figure $1 \mathrm{G}$ and $\mathrm{H}$ ).

Cerebrospinal fluid showed five cells with normal sugars (61 $\mathrm{mg} / \mathrm{dL})$ and protein $(33 \mathrm{mg} / \mathrm{dL})$. Cerebrospinal fluid Gram stain, culture, Indian ink, cryptococcal antigen, and tubercular cartridge-based nuclei acid amplification test were negative. However, her CSF galactomannan (GM) increased (Supplemental Table S1). The imaging picture and the raised CSF GM raised the suspicion of central nervous system (CNS) aspergillosis, hence started on oral voriconazole $200 \mathrm{mg} \mathrm{BD}$, and ATT was stopped.

She remained clinically stable. Imaging after a month of antifungal treatment showed further increase in the extent of signal changes into the left frontal lobe white matter (Figure 11 and J), with increased multiple foci of iso-intense signal (arrows in Figure 1J) in T2 hyperintense lesion. Enhancement pattern changed from nodular to punctate and linear with an increase in the extent of enhancement, especially in the left cerebral hemisphere (Figure $1 \mathrm{~K}$ and $\mathrm{L}$ ).

Despite 1 month of antifungal treatment, the brain lesions had increased in extent with an increase in the iso-intense foci on T2 and change in enhancement pattern to punctate and linear. Although nonspecific, T2 and Fluid-Attenuated Inversion Recovery (FLAIR) high-intensity lesions in the white matter are very common in CNS vasculitis, especially in primary angiitis. In current MRI, the iso-intense foci on T2 had significantly increased (arrows in Figure 1J), even to the 

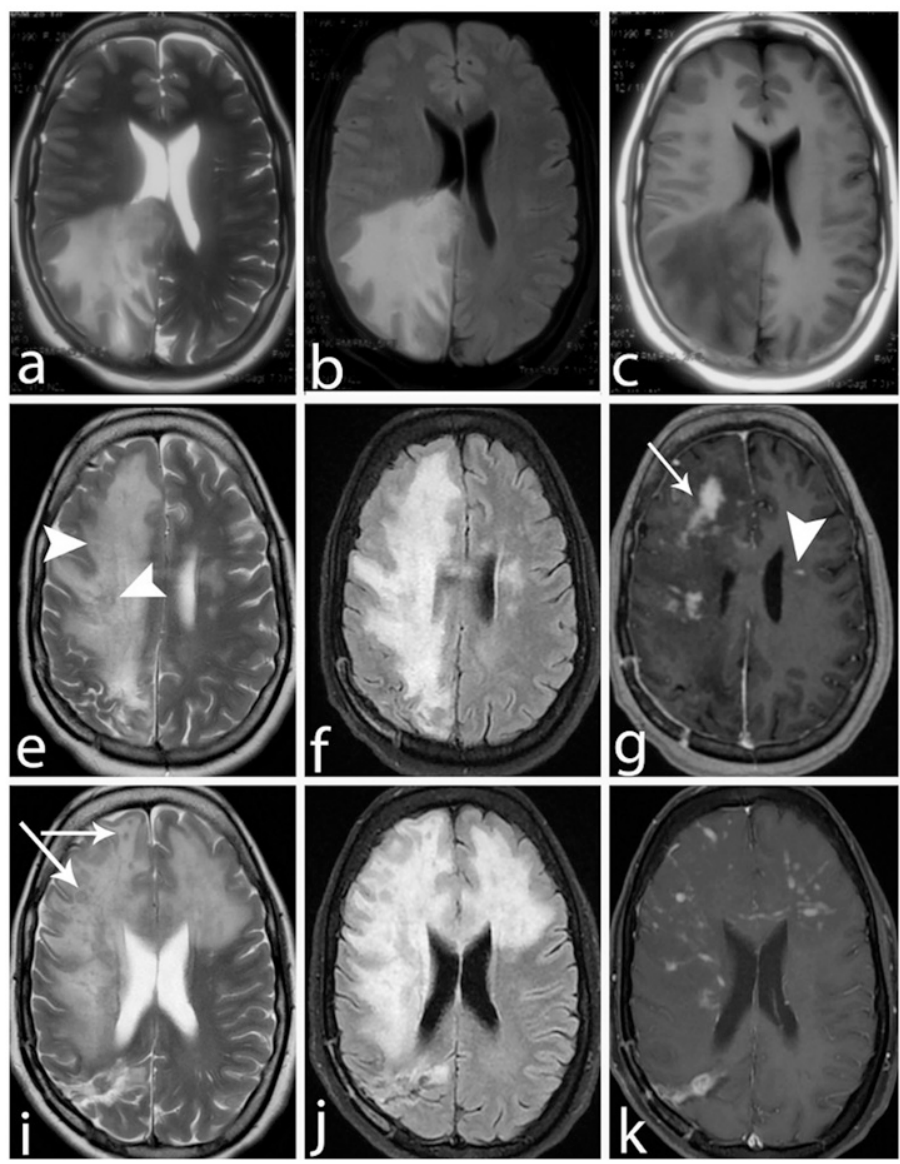

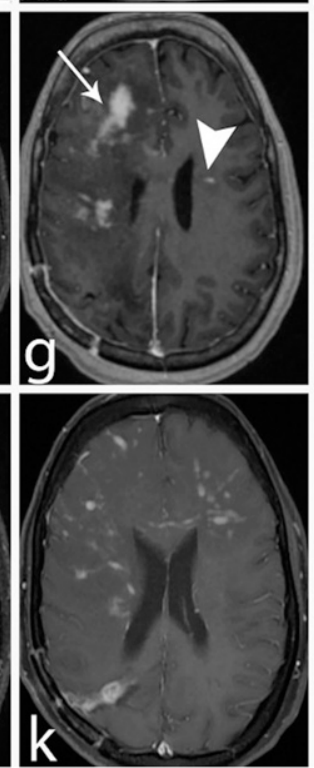

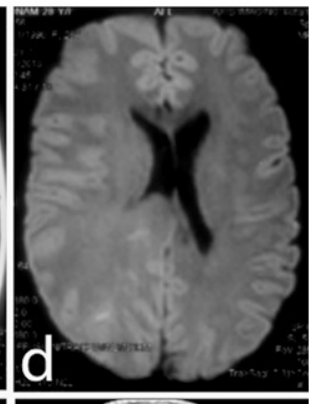
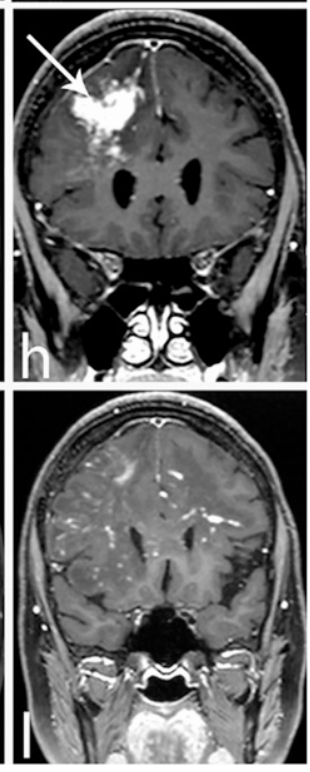

FIGURE 1. Initial MRI brain study. Magnetic resonance imaging of the brain shows a well-defined white matter lesion with mass effect in the right parieto-occipital region. The lesion is hyperintense on T2-WI (A) and FLAIR images (B), iso-hypointense on T1-WI (C), without any evidence of diffusion restriction in Diffusion-weighted image images (D). Follow-up MRI performed after 4 months (post-first biopsy) shows an increase in extent to white matter hyperintensity in T2-WI (E) and FLAIR images (F) to the right anterior frontal lobe and left corona radiata (arrowhead). Multiple isointense foci are seen within T2-hyperintense lesion (arrowhead in E). Patchy nodular enhancement is seen in the right frontal lobe (arrows in $\mathbf{G}$ and $\mathbf{H}$ ) and left centrum semiovale (arrowhead in $\mathbf{G}$ ) in axial $(\mathbf{G})$ and coronal $(\mathbf{H})$ post-gad T1-WI. Another follow-up MRI performed after a month of antifungal treatment shows further increase in the extent of signal changes into the left frontal lobe white matter in T2-WI (I) and FLAIR images (J). Multiple foci of iso-intense signal (arrows in $\mathbf{J}$ ) in T2 hyperintense lesion have also increased. Axial (K) and coronal (I) post-gad T1-WI change in enhancement pattern from nodular to punctate and linear and increase in extent of enhancement especially in the left cerebral hemisphere. MRI $=$ magnetic resonance imaging; $\mathrm{WI}=$ weighted image.

opposite hemisphere. In view of this and a noninfective CSF picture, CNS vasculitis was considered. An exhaustive workup (Supplemental Table S1) for secondary causes of vasculitis was inconclusive; hence, she was provisionally diagnosed as a case of primary angiitis of CNS.

She was given intravenous methylprednisolone $1 \mathrm{~g}$ daily for 5 days, followed by oral steroids. Magnetic resonance imaging after 1 month of steroid treatment showed a decrease in the extent of signal change (Figure $2 A$ and $B$ ), with ventricular and sulcal dilation suggesting volume loss. A decrease in linear and punctate enhancement was also noted (Figure $2 \mathrm{C}$ and $\mathrm{D}$ ). She was discharged on oral steroids and levetiracetam with a plan for further immunosuppression after 1 month. Clinically, there were no additional neurological deficits.

After 3 weeks, she reported back with a 5-day history of high-grade fever, headache, and altered sensorium. On examination, the patient was febrile, and disoriented with left hemiparesis and bilateral extensor plantar. Magnetic resonance imaging of the brain showed an increase in white matter T2-hyperintensity (Figure 2E) with sulcal effacement with multiple foci of diffusion restriction (arrows in Figure 2F).
Multiple ring-enhancing lesions in the bilateral frontal lobes $(R>L)$ and corpus callosum with localized mass effect were also noted (Figure $2 \mathrm{G}$ and $\mathrm{H}$ ).

The appearance of multiple randomly distributed ringenhancing lesions in MRI with striking high intensity on Diffusion-weighted image (DWI) (Figure 2F) suggested CNS Aspergillus abscesses. Cerebrospinal fluid study revealed 30 cells (100\% lymphocytes), normal sugars and raised protein (77 mg/dL), positive GM (1.32), and sterile cultures. She was started on intravenous amphotericin B $1.5 \mathrm{mg} / \mathrm{kg} /$ day. However, because of diagnostic uncertainty, a repeat brain biopsy was planned. Pus was found in the operative field. Histopathology of biopsy suggested an abscess cavity (Figure 3A-D). Gram stain of the operative specimens (pus) showed Gramnegative bacteria with safety pin appearance (Figure $3 \mathrm{E}$ ) and VITEK matrix-assisted laser desorption ionization timeof-flight mass spectrometry (VITEK MALDI-TOF MS database version 3.2) performed on the colonies that appeared on MacConkey, and blood agar revealed the organism as Burkholderia pseudomallei. But due to concern regarding missed identification and underrepresentation of this rare 

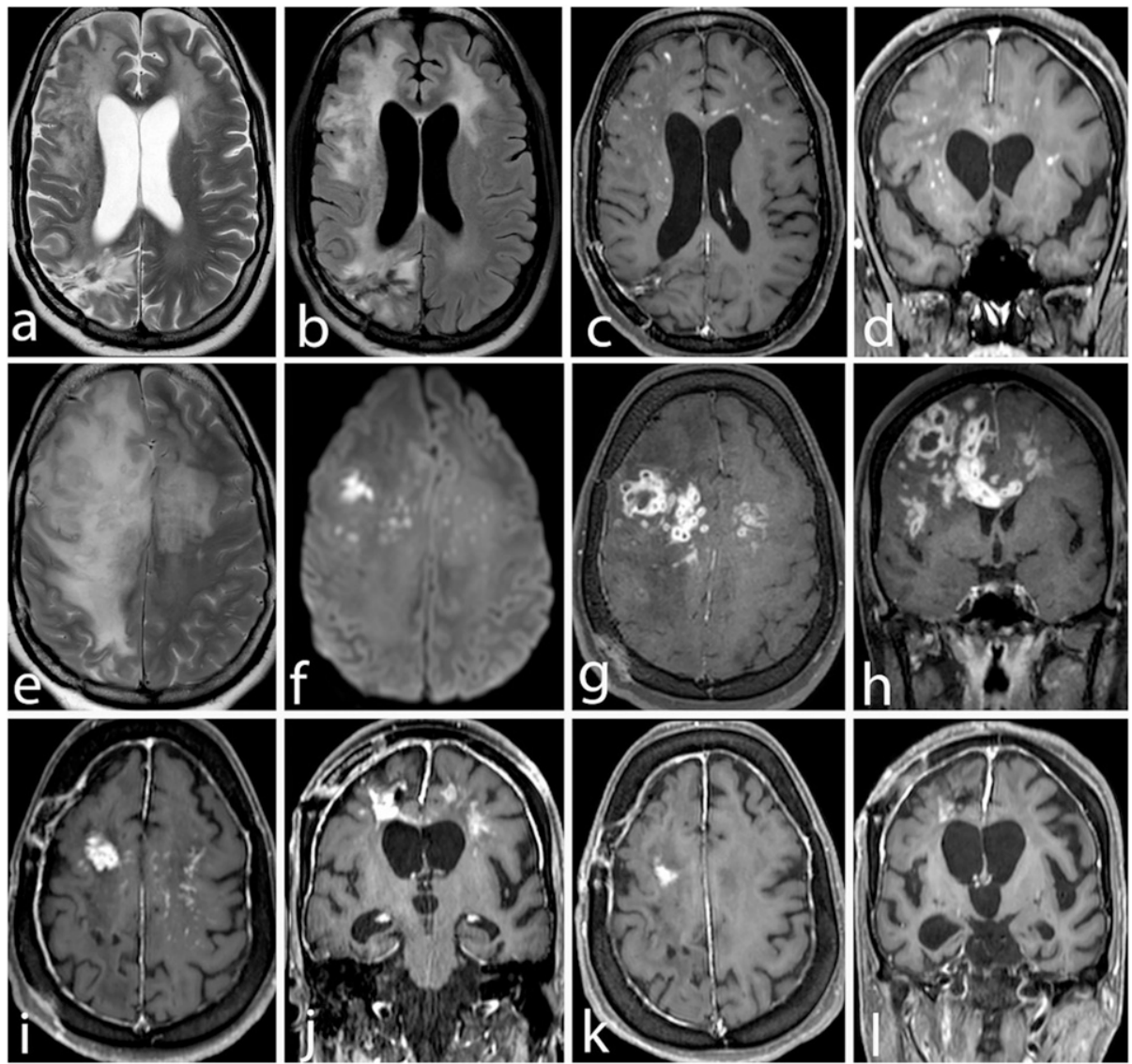

FIGURE 2. Follow-up MRI brain study. Follow-up MRI performed after 1 month of steroid treatment shows decrease in extent of signal change in T2-WI (A) and FLAIR images (B), with ventricular and sulcal dilation suggesting volume loss. Axial (C) and coronal (D) post-gad T1-WI shows decrease in linear and punctate enhancement. Patient was continued on steroids, and follow-up MRI performed after 3 weeks showed an increase in white matter T2-hyperintensity (E) with sulcal effacement. Diffusion-weighted image (F) shows multiple foci of diffusion restriction (arrows in $\mathbf{F})$. Contrast-enhanced axial $(\mathbf{G})$ and coronal $(\mathbf{H})$ T1-Wls show multiple ring-enhancing lesions in the bilateral frontal lobe $(R>L)$ and corpus callosum with localized mass effect. Axial (I) and coronal ( $\mathbf{J})$ post-gad T1-WI performed after 3 months of follow-up shows residual nodular and punctate enhancement with ventricular dilation. Repeat MRI after another 4 months shows significant reduction in enhancement with evidence of volume loss in post-gad axial (K) and coronal (L) T1-WI MRI. MRI = magnetic resonance imaging; $\mathbf{W I}=$ weighted image.

pathogen, we confirmed it by putting the VITEK 2 Gramnegative ID (BioMerieux) which confirmed the identification as $B$. pseudomallei.

Finally, a biopsy culture-proven diagnosis of CNS melioidosis explained the diagnostic confusion along the clinical course that was compounded by previous sterile CSF and biopsy cultures and raised CSF GM on two occasions and multiple empirical antibiotics and ATT that modified the clinical course. Although CSF GM antigen test is included in the revised European Organization for Research and Treatment of Cancer/Invasive Infectious Diseases Study Mycoses Group criteria for the diagnosis of cerebral aspergillosis (CA), ${ }^{1}$ only three studies have reported on GM antigen testing in CSF in a total of 27 patients with CA. In the study by Chong et al., ${ }^{2}$ it was shown that testing GM in CSF had a high diagnostic performance (sensitivity, specificity, and positive and negative predictive values were $88.2 \%, 96.3 \%, 93.8 \%$, and $92.9 \%$, respectively, when GM Optical Density Index cutoff of 1.0 was used) for diagnosing CA and may be useful to diagnose or virtually rule out $C A$ without the need for a cerebral biopsy. On the contrary, false positivity of GM assay may result from multiple other factors such as fungi Aspergillus (Fusarium, Penicillium, Cryptococcus, etc.), bacteria (Staphylococcus epidermidis, Corynebacterium, Escherichia, etc.), and crossreactivity with other antimicrobial agents (especially antibiotics containing tazobactam and clavulanate). ${ }^{3}$ Hence, CSF GM antigen testing may be used as a corroborative test for diagnosis or refuting CA rather than a definite test. Moreover, the prominent iso-intense signals on T2 led to a diagnosis of CNS vasculitis, which in retrospect, we interpreted as vasculitis secondary to the infectious process. High-dose steroids worsened her condition.

Antibiotic susceptibility testing of the operative pus showed sensitivity to ceftazidime, meropenem, minocycline, and trimethoprim-sulfamethoxazole. Amphotericin B was stopped and intravenous meropenem $1.0 \mathrm{~g}$ was given thrice daily and intravenous ceftazidime $2.0 \mathrm{~g}$ thrice daily. Meanwhile, her motor response deteriorated to $\mathrm{M} 3$, and she had recurrent generalized seizures, which were controlled with anesthetic infusions and antiepileptic drugs. She was intubated and subsequently tracheostomized.

Screening for any other systemic involvement of melioidosis was negative. Meropenem and ceftazidime were continued for a total of 12 weeks as per consensus guidelines. ${ }^{4,5}$ Her motor response improved to M5 (localizing to painful stimulus) on Glasglow Coma Scale (GCS). However, she 


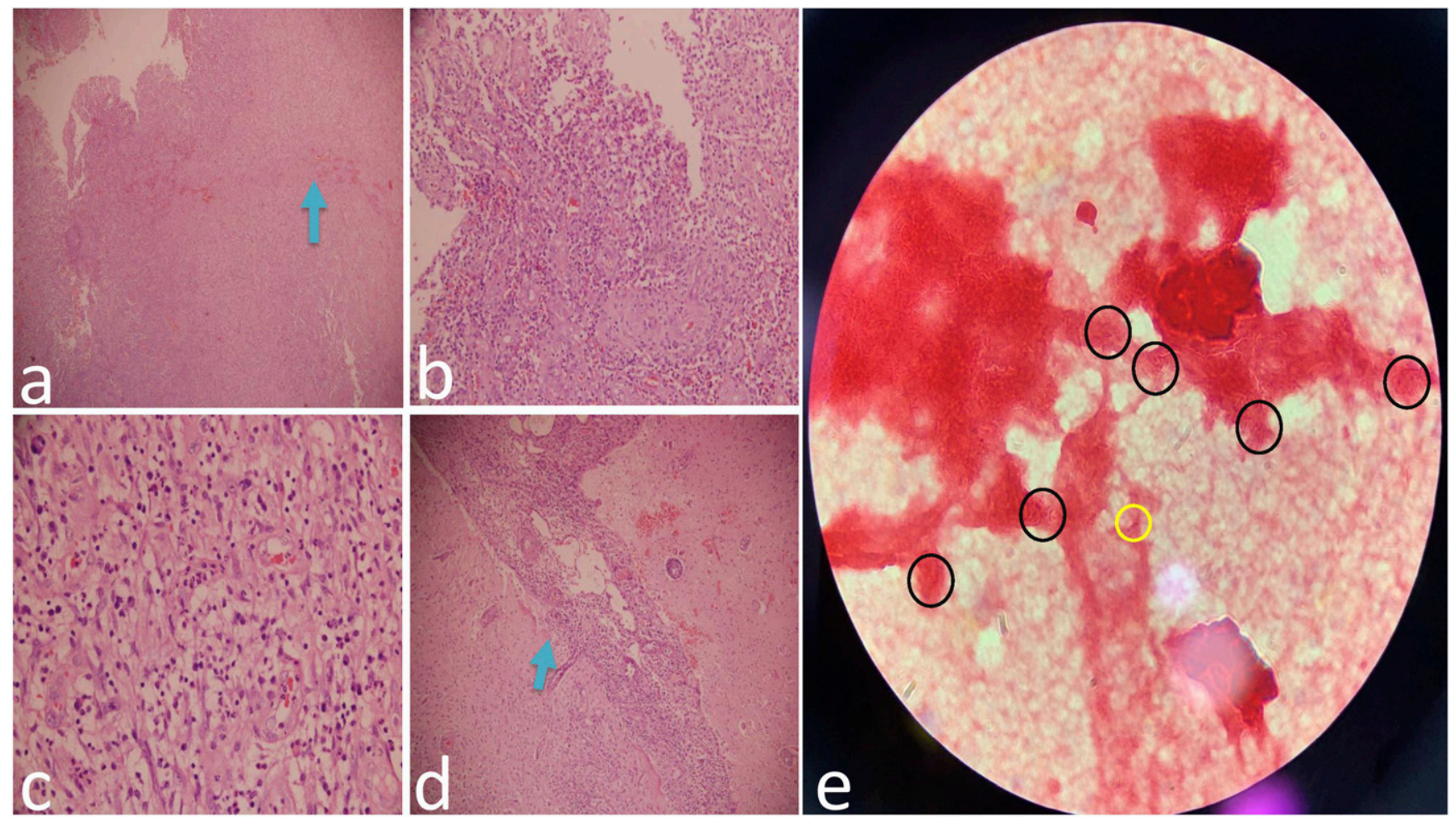

FIGURE 3. Histopathological images and Gram staining of the brain biopsy tissue. Photomicrographs showing the wall of an abscess (A, hematoxylin and eosin stain [H\&E] $\times 40$ ); low power demonstrating admixture of vascular proliferation and inflammatory cells $(\mathbf{B}, \mathrm{H} \& \mathrm{E} \times 100)$; inflammatory cells are an admixture of neutrophils and lymphocytes $(\mathbf{C}, \mathrm{H} \& \mathrm{E} \times 400)$. At places, inflammatory cells are spreading to subarachnoid space (D, H\&E × 100). Gram stain showing (E) Gram-negative bacteria in safety pin appearance (yellow circle), and black circles are showing masses of the same bacterium $(\mathrm{E})$, which ultimately grew Burkholderia pseudomallei in culture. This figure appears in color at www.ajtmh.org.

remained bedridden and needed day-care assistance. Followup MRI after 3 months showed residual nodular and punctate enhancement with ventricular dilation (Figure $2 \mathrm{l}$ and J). She was discharged on doxycycline $100 \mathrm{mg}$ twice daily, levetiracetam $1.0 \mathrm{~g} \mathrm{BD}$, clobazam $10 \mathrm{mg} \mathrm{BD}$, and lacosamide $100 \mathrm{mg}$ BD. At the time of discharge she was modified Rankin Score(mRS) 5, had spastic quadriparesis, left more than right, bedridden and needed day-care nursing and assistance.

Follow-up MRI after another 4 months showed a significant reduction in enhancement with evidence of volume loss (Figure $2 \mathrm{~K}$ and $\mathrm{L}$ ). She completed 6 months of doxycycline $100 \mathrm{mg}$ twice daily (eradication phase). At 1-year follow-up, the patient was still mRS five and was on antiepileptics and supportive therapy. Now, she occasionally smiles at her relatives, makes incomprehensible sounds (tracheostomy decannulated), seems to follow conversations, and spontaneously moves her right upper and lower limbs.

\section{DISCUSSION}

Central nervous system melioidosis, commonly encountered in the endemic areas, ${ }^{6,7}$ is caused by the Gram-negative bacterium $B$. pseudomallei. Central nervous system is affected in about $3 \%$ of melioidosis cases, ${ }^{8}$ and is more common in patients with a previous comorbidity ${ }^{9}$ and carries high mortality $(20 \%){ }^{9}$ Melioidosis occurs predominantly in northern Australia, Southeast Asia, South Asia (including India), and China. Most of the diagnosed cases are from Thailand, Malaysia, Singapore, and northern Australia. ${ }^{10}$ Northeastern
Thailand and parts of northern Australia are "hyperendemic" for melioidosis. ${ }^{9}$ In India, the southern states, particularly Karnataka and Tamil Nadu, are endemic for melioidosis. ${ }^{11}$ Sood et al. ${ }^{12}$ had described a case of septicaemia due to B. pseudomallei from Rajasthan. Garg et al. ${ }^{13}$ had described two cases of melioidosis in north India, one with liver abscess and another with brain abscess. Apart from these, there are no other reported cases in areas near the present case. A retrospective travel history also found no travel outside of Rajasthan. Recently, Wongwandee and Linasmita ${ }^{9}$ did a systematic review of all the case series and case reports, inclusive of all age-groups. Supplemental Table S2 shows a comparative predominant clinical, radiological, and pathological feature of the cases reported and the present case. In the present case, the diagnosis was delayed as the initial imaging was initially attributed to tuberculosis and later to aspergillosis and CNS vasculitis. Also, she did not belong to an endemic area and had no comorbidities. The radiological picture in CNS melioidosis is known to simulate CNS malignancy ${ }^{14}$ and tuberculomas. ${ }^{15}$ However, in the present case, the presence of multiple conglomerated enhancing lesions raised the suspicion of fungal etiology initially, and later when the response to antifungals was inadequate, the prominent iso-intense signals on T2 sequence led to suspicion of CNS vasculitis. Most CNS melioidosis present as encephalomyelitis and brain abscess and commonly involve the frontal lobe and brain stem. ${ }^{9}$ In most CNS melioidosis, the diagnosis was established with positive blood culture, which was again not forthcoming in the present case. Finally, when the diagnosis was ascertained with culture 
from brain tissue biopsy, appropriate treatment was instituted, followed by a proper duration of eradication therapy. The recommended treatment duration is usually a curative phase of intravenous antibiotics (Supplemental Table S2) for 68 weeks, followed by at least 4-6 months of eradication therapy. ${ }^{16,4}$ Despite the considerable delay in diagnosis and the inherent high mortality rate associated with this disease, the patient survived, but she lived with substantial morbidity. This case highlights the importance of awareness of this rare CNS infectious disease among physicians, even in nonendemic areas and healthy individuals, as the radiological and clinical features can masquerade several other disorders, and a high degree of suspicion can lead to early diagnosis, better outcome, and avoid mortality.

Received September 10, 2020. Accepted for publication November 25, 2020.

Published online January 11, 2021.

Note: Supplemental tables appear at www.ajtmh.org.

Acknowledgment: The American Society of Tropical Medicine and Hygiene (ASTMH) assisted with publication expenses.

Authors' addresses: Biswamohan Mishra, Venugopalan Y. Vishnu, Rohit Bhatia, Mamta Bhushan Singh, Roopa Rajan, Anu Gupta, and Madakasira Vasantha Padma Srivastava, Department of Neurology, All India Institute of Medical Sciences, New Delhi, India, E-mails: biswamohan26@gmail.com, vishnuvy16@yahoo.com, rohitbhatia71@yahoo.com, mbsneuro@gmail.com, rooparajan@ aiims.edu, doctoranugupta1@gmail.com, and vasanthapadma123@ gmail.com. Ajay Garg, Department of Neuroradiology, All India Institute of Medical Sciences, New Delhi, India, E-mail: drajaygarg@ gmail.com. Ramesh Sharanappa Doddamani and Parul Singh, Department of Neurosurgery, All India Institute of Medical Sciences, New Delhi, India, E-mails: drsdramesh@gmail.com and parul5fiore@ gmail.com. Mehar Chand Sharma, Department of NeuroPathology, All India Institute of Medical Sciences, New Delhi, India, E-mail: sharmamehar@yahoo.co.in.

\section{REFERENCES}

1. Donnelly JP et al., 2020. Revision and update of the consensus definitions of invasive fungal disease from the European organization for research and treatment of cancer and the Mycoses Study Group Education and Research Consortium. Clin Infect Dis 71: 1367-1376.
2. Chong GM, Maertens JA, Lagrou K, Driessen GJ, Cornelissen JJ, Rijnders BJA, 2016. Diagnostic performance of galactomannan antigen testing in cerebrospinal fluid. J Clin Microbiol 54: 428-431.

3. Dixon C, 2011, Detection of Galactomannan in Broncho-Alveolar Lavage Fluids by Platelia Aspergillus Enzyme Immunoassay, Division of Special Pathogen and Transplant Products, Mycoses Study Group, Biorad Laboratories, USA and Sanofi Diagnostics, France.

4. Lipsitz R et al., 2012. Workshop on treatment of and postexposure prophylaxis for Burkholderia pseudomallei and B. mallei infection, 2010. Emerg Infect Dis 18: e2.

5. Pitman MC, Luck T, Marshall CS, Anstey NM, Ward L, Currie BJ, 2015. Intravenous therapy duration and outcomes in melioidosis: a new treatment paradigm. PLoS Negl Trop Dis 9: e0003586.

6. Currie BJ et al., 2000. Endemic melioidosis in tropical northern Australia: a 10-year prospective study and review of the literature. Clin Infect Dis 31: 981-986.

7. Kingsley PV, Leader M, Nagodawithana NS, Tipre M, Sathiakumar N, 2016. Melioidosis in Malaysia: a review of case reports. PLoS Negl Trop Dis 10: e0005182.

8. Currie BJ, Ward L, Cheng AC, 2010. The epidemiology and clinical spectrum of melioidosis: 540 cases from the 20 year Darwin prospective study. PLoS Negl Trop Dis 4: e900.

9. Wongwandee M, Linasmita $P, 2019$. Central nervous system melioidosis: a systematic review of individual participant data of case reports and case series. PLoS Negl Trop Dis 13: e0007320.

10. Wiersinga WJ, Virk HS, Torres AG, Currie BJ, Peacock SJ, Dance DAB, Limmathurotsakul D, 2018. Melioidosis. Nat Rev Dis Primers 4: 17107.

11. Mukhopadhyay C, Shaw T, Varghese GM, Dance DAB, 2018. Melioidosis in South Asia (India, Nepal, Pakistan, Bhutan and Afghanistan). Trop Med Infect Dis 3: 51.

12. Sood S, Khedar RS, Joad SH, Gupta R, 2014. Septicaemic melioidosis: case report from a non-endemic area. J Clin Diagn Res 8: DD01-DD02.

13. Garg G, Chawla N, Chawla K, Khosla P, Jain S, 2015. Atypical presentations of melioidosis in north India: report of two cases. $J$ Assoc Physicians India 63: 82-83.

14. Huang W, Wu G, Chen F, Li M, Li J, 2018. Multi-systemic melioidosis: a clinical, neurological, and radiological case study from Hainan province, China. BMC Infect Dis 18: 649.

15. Vidyalakshmi K, Lipika S, Vishal S, Damodar S, Chakrapani M, 2012. Emerging clinico-epidemiological trends in melioidosis: analysis of 95 cases from western coastal India. Int $J$ Infect Dis 16: e491-e497.

16. Dance D, 2014. Treatment and prophylaxis of melioidosis. Int $J$ Antimicrob Agents 43: 310-318. 